\title{
An Early Prediction of the Amplitude of Solar Cycle 25
}

\author{
W. Dean Pesnell ${ }^{1}$ Kenneth H. Schatten ${ }^{2}$
}

Received: 3 December 2017 / Accepted: 11 July 2018 / Published online: 27 July 2018

(C) This is a U.S. government work and not under copyright protection in the U.S.; foreign copyright protection may apply 2018

\begin{abstract}
A "Solar Dynamo" (SODA) Index prediction of the amplitude of Solar Cycle 25 is described. The SODA Index combines values of the solar polar magnetic field and the solar spectral irradiance at $10.7 \mathrm{~cm}$ to create a precursor of future solar activity. The result is an envelope of solar activity that minimizes the 11-year period of the sunspot cycle. We show that the variation in time of the SODA Index is similar to several wavelet transforms of the solar spectral irradiance at $10.7 \mathrm{~cm}$. Polar field predictions for Solar Cycles $21-$ 24 are used to show the success of the polar field precursor in previous sunspot cycles. Using the present value of the SODA index, we estimate that the next cycle's smoothed peak activity will be about $140 \pm 30$ solar flux units for the $10.7 \mathrm{~cm}$ radio flux and a Version 2 sunspot number of $135 \pm 25$. This suggests that Solar Cycle 25 will be comparable to Solar Cycle 24. The estimated peak is expected to occur near $2025.2 \pm 1.5$ year. Because the current approach uses data prior to solar minimum, these estimates may improve as the upcoming solar minimum draws closer.
\end{abstract}

Keywords Solar Cycle · Predictions

\section{Long Term Solar Activity Forecasting Techniques}

Over the past five sunspot cycles, a growing group of solar forecasters have used a variety of methods to produce long-term predictions of solar activity. This effort produced at least 105 predictions of the amplitude of Solar Cycle 24 (Pesnell, 2016). These solar activity prediction methods are especially important for NASA and other agencies interested in low altitude satellite orbits because the lifetime and orbital trajectory parameters of satellites in low terrestrial or planetary orbits are greatly affected by the varying solar fluxes.

\footnotetext{
$凶$ W.D. Pesnell

William.D.Pesnell@NASA.gov

K.H. Schatten

solarcycleken@gmail.com

1 Solar Physics Laboratory, NASA/Goddard Space Flight Center, Greenbelt, MD, USA

2 a.i. solutions, Lanham, MD, USA
} 
Other space-weather effects, such as ground-induced currents that cause power outages and radiation doses in flight crews, can be anticipated using these predictions.

About $1 / 3$ of those predictions relied on a precursor, where another variable is used to track solar activity through solar minimum. Some use the solar polar magnetic field as that variable. Others use geomagnetic activity as the precursor. Precursors have tended to perform well as predictors of solar activity.

Geomagnetic precursor methods were introduced by Ohl and Ohl (1979) and Brown and Williams (1969), who noticed that fluctuations in the Earth's magnetic field caused by geomagnetic activity could be used to predict solar activity. As space-based assets grew in importance, predictions of the level of activity in the next cycle were required at ever-earlier phases of the current sunspot cycle. This led to efforts to remove the solar activity component of geomagnetic activity from the geomagnetic indices (Feynman, 1982; Hathaway, 2010). Until very close to the solar minimum in December 2008, when the recurrent activity traceable to the polar coronal hole and related equatorial extensions thereof dominates the solar magnetic field striking the Earth, this correction produced wide variations in the geomagnetic precursor predictions of Solar Cycle 24 (Pesnell, 2014).

Fewer predictions have been made using solar polar magnetic field precursors-only two for Solar Cycle 24 (Schatten, 2005; Svalgaard, Cliver, and Kamide, 2005). Both were for low activity in Solar Cycle 24, which was realized when the cycle peaked at a sunspot number of about 80 in 2014. This prediction relies on the most basic and robust aspect of dynamo theory: that future magnetic field develops from pre-existing magnetic field.

By contrast, a large group of predictions searches for statistical correlations within the sunspot number series. This class of predictions had a poor record of predicting the amplitude of Solar Cycles 21-24. Pesnell (2016) showed that the skill scores of these "climatological" predictions mimicked the long-term average in their ability to make a forecast of Solar Cycle 24. Brown (1986), Hathaway, Wilson, and Reichman (1999), and Li, Yun, and $\mathrm{Gu}$ (2001) showed that the statistical class of predictions was less accurate than the precursor class for the last few sunspot cycles. The continuing popularity of statistical predictions may be the ease of seeking out correlations that sample the time series and its inherent noise properties in novel but unphysical ways.

The solar polar field precursor can be developed into the "dynamo method", which provides a physical foundation for that precursor method (Schatten and Pesnell, 1993). From our understanding of the physical mechanisms of the Sun's dynamo, we can then understand how the solar wind drives the terrestrial geomagnetic field and allows geomagnetic field to serve as a precursor for future solar activity. Let us first discuss the solar dynamo, then the dynamo method of solar activity forecasting, how well those predictions fared in Solar Cycles 21-24, and finally a prediction of the amplitude of Solar Cycle 25.

\section{The Solar Dynamo}

Descriptions of the solar dynamo can be found in Waldmeier $(1939,1955)$, Babcock (1961), Leighton (1969), Parker (1977), Howard (1977), Sheeley, Wang, and Harvey (1989), and Charbonneau (2010). These are becoming more faithful representations by including current data in the models to drive the models to the present Sun (Muñoz-Jaramillo, Nandy, and Martens, 2009). Although the workings of the solar dynamo are generally understood, the details are shrouded by a photospheric "cloak" covering the Sun. Recent helioseismic studies have looked below that cloak to reveal the plasma flows, both shallow (Zhao, Kosovichev, 


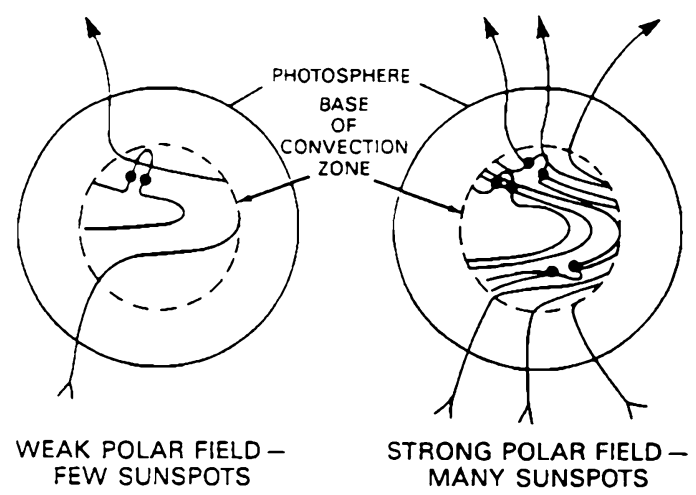

Figure 1 Two composite drawings that combine the polar fields at the start of the cycle with the toroidal field in the form of sunspots near solar maximum to illustrate how the dynamo method works (Schatten and Pesnell, 1993). The left panel shows a relatively weak sunspot cycle, while the right shows a more active sunspot cycle. The weak cycle (left panel) illustrates that when the polar fields are weak, few sunspots will form later in the cycle, and the right panel displays the opposite effect.

and Bogart, 2014) and deep (Zhao et al., 2013), that generate, amplify, and transport the solar magnetic field within the solar convection zone.

Fortunately, the dynamo method of solar cycle prediction depends only upon the most basic and robust aspect of dynamo theory: that future magnetic field develops from preexisting magnetic field. This provides a certain persistence in the long-term behavior of solar magnetic fields that dynamo prediction methods utilize. Evidence for this persistence may be found in the $80-100$ year timeframe of the Gleissberg cycle.

Let us now consider how the dynamo method of solar activity prediction works. We assume that the Sun's polar field at the start of a cycle is transformed by dynamo processes into a toroidal field within the Sun, appearing years afterwards during the maximum phase. The Sun's polar field then serves as a "seed" for future solar activity; and it can be used to forecast future activity. In the dynamo method (Schatten et al., 1978), one uses the Sun's polar fields at solar minimum to estimate future solar activity. Figure 1 illustrates the field geometry involved in the solar dynamo method (with both the polar fields and the toroidal fields portrayed in the same view although they peak at separate times). For a solar cycle when the polar field is weak, little toroidal field is generated, and few sunspots develop. In another cycle, when the polar field is strong, the reverse is true. Elementary dynamo theory suggests that a linear relationship exists to lowest order.

\section{Data Sources and Correlation Fits Between $R_{Z}$ and F10.7}

Our research requires estimates of the solar magnetic field during both the maxima of solar activity and the minima. We use the solar spectral irradiance at $10.7 \mathrm{~cm}(\mathrm{~F} 10.7$, reported in units of $10^{-22} \mathrm{~W} \mathrm{~m}^{-2} \mathrm{~Hz}^{-1}$ ) as our primary index of solar activity. F10.7 has been measured since 1947 (Tapping, 2013). Because it is available soon after each days' measurement, it is commonly used in atmospheric drag calculations. Adjusted F10.7 (normalized to 1 AU) are used in this analysis, although the "calibrated" version, where each adjusted value was multiplied by 0.9 , was used in some earlier work.

Another well-known index of solar activity is the sunspot number, which is reported daily as the International Sunspot Number $\left(R_{Z}\right)$. There are two versions of $R_{Z}$, the original 
(Version $1, R_{Z}^{V_{1}}$ ) and the new Version $2\left(R_{Z}^{V_{2}}\right)$ introduced by Clette $e$ t al. (2014), which has been the official version since 1 June 2015 .

F10.7 peaks near solar maximum and drops to a value of about 60 at solar minimum. While F10.7 is well-correlated with both $R_{Z}^{V_{1}}$ and $R_{Z}^{V_{2}}$, there were significant differences between F10.7 and $R_{Z}^{V_{1}}$ that are reduced in $R_{Z}^{V_{2}}$. These discrepancies are discussed in Clette et al. (2014).

Linear correlation fits to convert the adjusted F10.7 to both calibrations of the sunspot number and vice versa were calculated for this research. Monthly averages of the datasets were used in a linear fit that allows for errors in both variables.

- For times since $1950, R_{Z}^{V_{2}}=0.246+1.41 R_{Z}^{V_{1}}$.

- For times since $1955, \mathrm{~F} 10.7=62( \pm 3)+0.648( \pm 0.025) R_{Z}^{V_{2}}$.

- For times since $1955, \mathrm{~F} 10.7=61.6+0.920 R_{Z}^{V_{1}}$.

Because errors in both variables are accounted for in the analysis, the fits can be inverted. For example $R_{Z}^{V_{1}}=\left(R_{Z}^{V_{2}}-0.246\right) / 1.41$ to the same accuracy as the reciprocal fit.

\subsection{Solar Polar Field}

Our primary measure of the solar magnetic field at solar minimum is the polar magnetic field $\left(B_{p o l}\right)$, which has been measured at the Wilcox Solar Observatory since 1975 (Hoeksema, 2010). We used the table of values on the WSO website, with values corrected for calibration issues with the polarimeter from 16 Dec. 2016 to 18 May 2018.

\section{The "Solar Dynamo Amplitude" (SODA) Index}

We generalized the dynamo method, so that it can be used at any phase of the solar cycle (Schatten and Pesnell, 1993). In previous work, we showed that the poloidal field at solar minimum could be used as an estimate of the toroidal field, the source of solar activity, at the next maximum. Thus, to ascertain how active a cycle we are experiencing, we can count the number of sunspots or use F10.7, etc. This is essentially what the McNish-Lincoln technique does (Suggs, 2013; McNish and Lincoln, 1949), but it also adds a measure of curve fitting to augment the values. We combine our previous work on polar fields with measurements of the toroidal fields that cause solar activity, using the well-known sunspot number or F10.7.

Over a solar cycle, the solar magnetic field oscillates between toroidal and poloidal components, akin to the variations of kinetic and potential energy in a simple harmonic oscillator. In the absence of secular variability, these forms can be scaled and added in quadrature to obtain an invariant of the system, or at least a slowly varying quantity.

The Sun also experiences short-term variations, such as bursts of activity, when many active regions manifest themselves; periods of quiescence; and secular changes. In the presence of solar secular variations, our index will not be constant. Our goal is to reduce the power at the 10-11 year period of the solar cycle, which would be most prominent if we were to monitor either the toroidal or poloidal component alone, and form a quantity which better represents the slowly varying envelope of the strength of the solar dynamo.

To this end, Schatten and Pesnell (1993) developed the "SOlar Dynamo's Amplitude" (SODA) Index, to provide a measure of the magnetic flux within the Sun's convection zone at any phase of the solar cycle. There are two forms of the SODA index. 
Figure 2 Shown are the last 43 years of F10.7, $\left|B_{\text {pol }}\right|$ from the Wilcox Solar Observatory (scaled by $162 / 1.28=127$ to be in units of F10.7), and the SODA index defined by Equation 1, in the same units as F10.7. Note that the SODA Index has reduced power at the 11 year period by combining the datasets, not by using a filter.

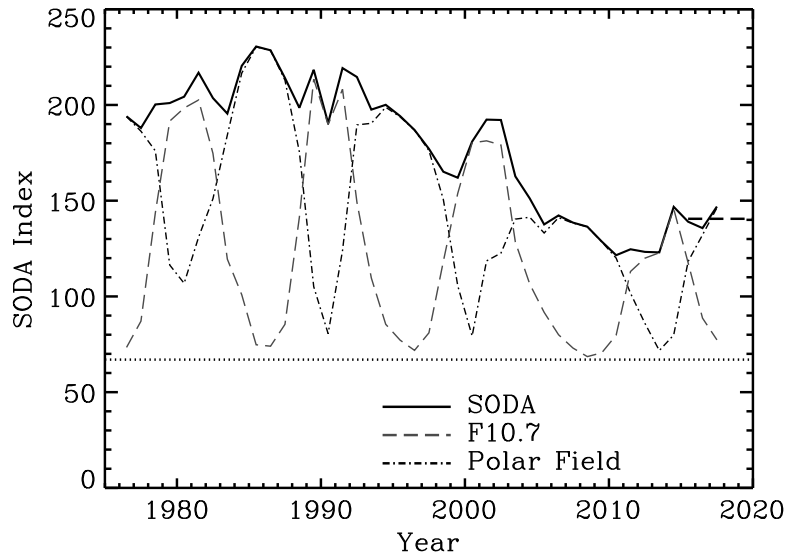

The SODA Index uses F10.7 as a measure of the Sun's toroidal field and direct observations of the Sun's polar fields from the Wilcox Solar Observatory as the poloidal field. The other index (the XSODA Index) uses the extensive historical dataset of sunspot number and polar faculae counts to cover almost a century of solar measurements, but will not be discussed further. We note that Priyal et al. (2014) describes a polar network index from Ca-K spectroheliograms that provides a measure of the solar polar magnetic field since 1904. This index may be usable in an extended SODA, much like polar faculae were used by Schatten and Pesnell (1993) in the XSODA Index. This may also be true for the polar faculae database described by Muñoz-Jaramillo et al. (2012).

Values of F10.7 and the averaged polar magnetic field ( $B_{p o l}$, in units of gauss) are smoothed with an annual or semiannual average. A straight annual average is used as the 13-month average did not change the results. The annual and semiannual results can then be more easily compared. Figure 2 illustrates how the SODA index combines the smoothed, averaged $B_{p o l}$ with the smoothed F10.7 through

$$
\mathrm{SODA}=67+162\left[\left(\frac{B_{\text {pol }}}{1.28}\right)^{2}+\left(\frac{\mathrm{F} 10.7-67}{162}\right)^{2}\right]^{1 / 2}
$$

where we have normalized the index to F10.7 units. Again, the scaling is chosen to optimally reduce the Fourier transform power at 11 years. The SODA index allows us to predict the maximum value of the next solar cycle prior to the maximum in the Sun's polar field. It does this by including the toroidal field that is fading from the previous cycle but that is expected to reappear as poloidal field at solar minimum, and subsequently as toroidal field during the maximum of the next cycle. Using a semiannual average gave the same predicted value but allows the SODA Index to be calculated closer to the present (90 days delayed vs. 183 days delayed for the annual average).

At solar maximum, the SODA index will simply use the maximum activity level in F10.7 to predict future levels. This "inertial" forecast is not very accurate (Pesnell, 2008). Between solar maximum and solar minimum, the polar field will become increasingly important in estimating future activity levels, and at solar minimum, it will use the polar field data alone. To predict the future level of F10.7, we use the average of the three most recent estimates of the SODA Index from Equation 1 above. 


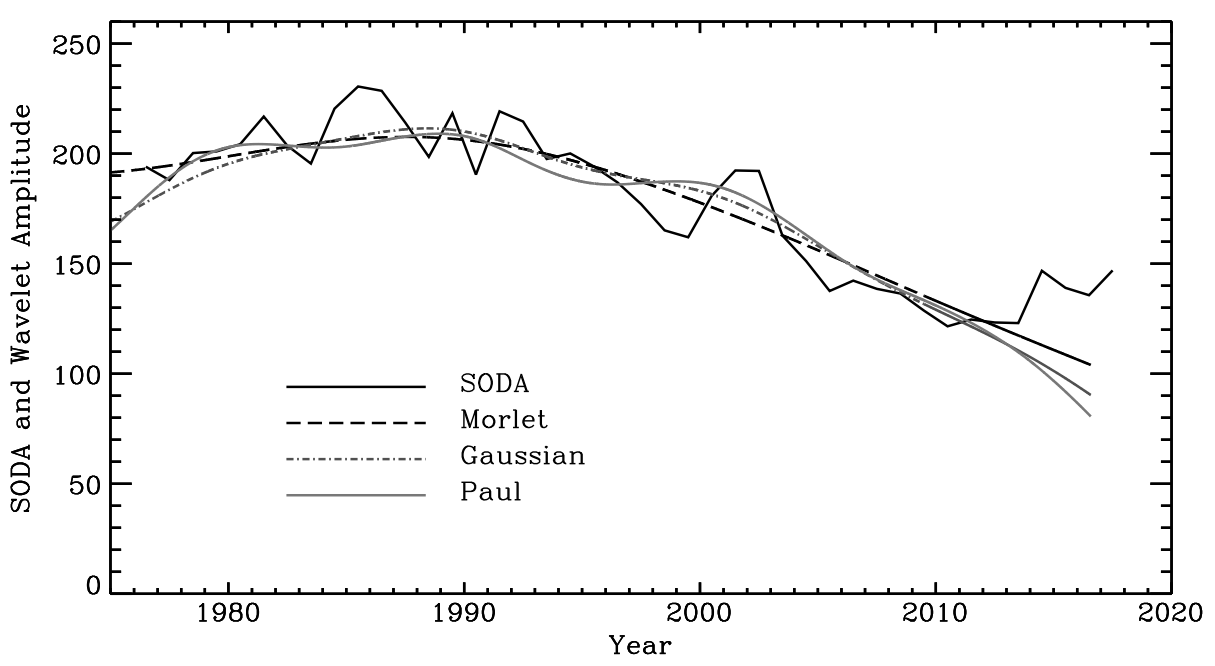

Figure 3 A comparison of the SODA Index from 1975 to the present with the square root of wavelet power summed over scale for three different wavelet transforms of F10.7. The SODA Index is shown as a black curve and the wavelet transforms as dashed curves of varying darkness. Dashed curves of the same darkness extend the wavelet transform amplitude into the zone of exclusion at a scale of 11 years for each wavelet.

\subsection{Comparison of SODA with Wavelet Amplitude}

A wavelet analysis can also provide an estimate for the envelope of solar activity. The wavelet power, summed over scale, tracks the evolution of the dominant 11-year period of solar activity. A comparison of the SODA Index to wavelet transforms of F10.7 would show how the SODA Index tracks the envelope of solar activity. For our purposes, the wavelet transforms were calculated using the Paul, Morlet, and derivative of Gaussian (DOG) mother wavelets. The DOG wavelet routine in IDL was modified to return the total power and to use a recursive calculation of the Hermite polynomials to extend the order to arbitrary values. All wavelets were calculated with an order parameter of eight, although the interpretation of the order varies somewhat from one mother wavelet to another. Figure 3 shows the square root of the wavelet transform power (i.e., the amplitude), summed over scale for all three mother wavelets. The amplitudes are very smooth because the 11-year period of the sunspot cycle dominates all of the other variations.

Figure 3 also shows how the SODA Index tracks the amplitude of the wavelet transform power, summed over scale for all three mother wavelets. The wavelet amplitude reached a broad local peak in the years before 1990 and has since generally decreased. Since 2010, the wavelet amplitude tends toward zero as the zone of exclusion for the dominant 11-year period of solar activity is entered and the wavelets no longer resolve that timescale. A similar behavior would be seen in left side of the plot if it extended back to the beginning of the F10.7 data. Because the SODA Index can be created annually or even semi-annually, it includes information from much closer to the present and continues at a larger value.

\section{Polar Field Predictions of Solar Cycles 21 - 24}

The various dynamo methods have correctly predicted, often several years in advance, the magnitude of Solar Cycles 21 - 24 (Schatten et al., 1978; Schatten and Sofia, 1987; Schatten, 
Figure 4 A plot of the solar spectral irradiance at $10.7 \mathrm{~cm}$ (F10.7) and predictions for Solar Cycles 21-25. The vertical lines are the publication dates of each prediction, which are also listed in Table 1. The solid black line is the annually averaged F10.7, the dashed grey are solar polar precursor and SODA predictions, and the error bars show the $\pm 1 \sigma$ limits (including the estimated timing uncertainty of 1.5 year). Each prediction is labeled with the Solar Cycle number.

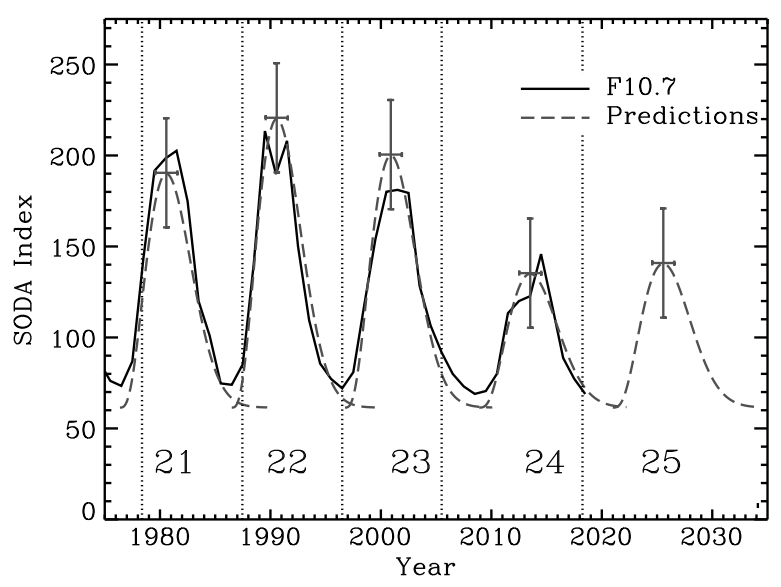

Table 1 Polar magnetic field predictions of solar activity. Note that the peak sunspot number forecast shown here for Cycle 25 was made using V1 sunspot number.

\begin{tabular}{llllll}
\hline Solar Cycle & Pred. $R_{Z}^{V_{1}}$ & Pred. F10.7 & Actual F10.7 & Pred. date $^{2}$ & Reference \\
\hline 21 & $140 \pm 20$ & 190 & 200 & 1978.4 & Schatten et al. (1978) \\
22 & $170 \pm 25$ & 220 & 213 & 1987.5 & Schatten and Sofia (1987) \\
23 & 150 & $200 \pm 30^{*}$ & 180 & 1996.2 & Schatten, Myers, and Sofia (1996) \\
24 & $80 \pm 30$ & $135 \pm 30^{*}$ & 146 & 2005.8 & Schatten (2005) \\
25 & $85 \pm 30$ & $140 \pm 30$ & & 2018.5 & Current work \\
\hline
\end{tabular}

Notes:

* This published value of F10.7 was converted from calibrated to adjusted.

${ }^{1}$ See Figure 4.

${ }^{2}$ Date prediction was published.

Myers, and Sofia, 1996; Schatten, 2005). The smoothed peak for F10.7 in Solar Cycle 22 was expected to be $220 \pm 25$. This prediction was made and published in early 1987, prior to a dramatic increase in solar activity. Solar Cycle 22 peaked with a value in F10.7 of 213, agreeing well with the prediction. Let us now see the success of predictions of other Solar cycles.

Table 1 lists the solar polar condition predictions of smoothed activity for Solar Cycles $21-24$. The columns are the Solar cycle number, predicted $R_{Z}$, predicted F10.7, actual F10.7, the publication date of the prediction, and the reference. The numbers with error bars were the predicted quantity, while the italicized predictions were derived from a correlation fit of $R_{Z}^{V_{1}}=1.087$ (F10.7 - 61.6), which comes from the correlation fit in Section 3. These predictions of past cycles are shown in Figure 4, along with the annual averaged F10.7.

Schatten (2005) used the SODA Index to predict the amplitude of Solar Cycle 24 would be $124 \pm 30$ in terms of smoothed calibrated F10.7, which corresponds to an adjusted F10.7 of $135,80 \pm 30$ in terms of smoothed international sunspot number $\left(R_{Z}^{V_{1}}\right)$, and 115 in the new version of the international sunspot number $\left(R_{Z}^{V_{2}}\right)$. The measured F10.7 reached $146 \mathrm{SFU}$ in 2014. 


\section{SODA Index Prediction of Solar Cycle 25}

The 2018.3 value of the SODA index is 140 corresponding to an F10.7 amplitude of Solar Cycle 25 of $140 \pm 30 \mathrm{SFU}$ and sunspot amplitude of $R_{Z}^{V_{2}}=135 \pm 25$ (which would be $85 \pm 30$ using $R_{z}^{V_{1}}, c f$. Table 1). The F10.7 amplitude (140) is obtained from an average of the three most recent values in Figure 2, and the uncertainty $( \pm 30)$ from the variance in the data. This suggests that Solar Cycle 25, will be comparable to or even larger than Solar Cycle 24. The late surge of polar magnetic field may be an indication that the decline in solar activity over the last few sunspot cycles is over. If true, this would show how solar activity signals an increase in the amplitude of solar activity. However, this surge in the southern hemisphere is larger than any other in the WSO record and should be better understood.

The estimated peak is expected to occur near 2025.2 \pm 1.5 year. Although different methods can establish this solar cycle timing, we prefer the method of Schatten (1990) which uses the latitudes of solar active regions (the Butterfly diagram). At this time there no significant active regions have appeared and the timing reduces to adding the climatological average of $10.9 \pm 1.5$ years to the date of the previous maximum (2014.25) with an error estimate of the standard deviation of the previously measured cycle lengths from that average.

Because this early prediction technique is before solar minimum, these estimates be should regarded as preliminary, and may improve as solar minimum develops. The SODA Index becomes a solar polar field precursor at solar minimum, but without the correlation fit that would introduce information from previous cycles.

The last blue curve of Figure 4 (labeled 25) shows this early estimate of future activity. The curves are obtained from the estimated amplitude (140) and the $\pm 1 \sigma$ curves uncertainty from the estimated uncertainty $( \pm 30)$. The curves are generated with an algorithm provided by Hathaway, Wilson, and Reichman (1994). For our purposes, the predicted variation of sunspot number (V1) was calculated and converted to F10.7 with the correlation relation listed above.

There are two possible reasons which suggest the peak of Solar Cycle 25 might exceed this early estimate: The Sun's polar field may continue to increase until solar minimum is reached and the SODA values in Figure 2 suggest an increasing trend. Thus this "early estimate" may be conservative, leaving open the possibility of a future increase, however, this is still the best estimate we can make on physical grounds at the present time.

This work illustrates the difficulty in long-term predictions when the data used in the analysis can change in calibration and definition. It emphasizes the need to clearly articulate what data is being used and providing access to that data for the reviewers and readers of the paper.

Acknowledgements This work was supported by NASA's Solar Dynamics Observatory. F10.7 values are courtesy of the Dominion Radio Astronomy Observatory. Adjusted F10.7 (normalized to 1 AU) were downloaded from the National Geophysical Data Center (NGDC, https://www.ngdc.noaa.gov/stp/space-weather/ solar-data/solar-features/solar-radio/noontime-flux/penticton/penticton_adjusted/listings/listing_drao_ noontime-flux-adjusted_daily.txt). The polar magnetic field data are from the Wilcox Solar Observatory (http:// wso.stanford.edu/Polar.html) and are courtesy of J. T. Hoeksema. The Wilcox Solar Observatory is currently supported by NASA. International Sunspot Numbers were obtained from the Solar Influences Data Center (SIDC) website, http://www.sidc.be/silso/DATA/dayssnv0.dat for $R_{Z}^{V_{1}}$ and http://www.sidc.be/silso/ DATA/SN_d_tot_V2.0.txt for $R_{Z}^{V_{2}}$.

Disclosure of Potential Conflicts of Interest The authors declare that they have no conflicts of interest. 


\section{References}

Babcock, H.W.: 1961, The topology of the Sun's magnetic field and the 22-year cycle. Astrophys. J. 133, 572. DOI. ADS.

Brown, G.: 1986, Working group "A” report: long-term solar activity predictions. In: Simon, P.A., Heckman, G., Shea, M.A. (eds.) Solar-Terrestrial Predictions, 1. ADS.

Brown, G.M., Williams, W.R.: 1969, Some properties of the day-to-day variability of Sq(H). Planet. Space Sci. 17, 455. DOI. ADS.

Charbonneau, P.: 2010, Dynamo models of the solar cycle. Living Rev. Solar Phys. 7(3). DOI.

Clette, F., Svalgaard, L., Vaquero, J.M., Cliver, E.W.: 2014, Revisiting the sunspot number. Space Sci. Rev. 186(1-4), 35. DOI.

Feynman, J.: 1982, Geomagnetic and solar wind cycles, 1900-1975. J. Geophys. Res. 87, 6153.

Hathaway, D.H.: 2010, The solar cycle. Living Rev. Solar Phys. 7(1). DOI.

Hathaway, D.H., Wilson, R.M., Reichman, E.J.: 1994, The shape of the sunspot cycle. Solar Phys. $151,177$. DOI.

Hathaway, D.H., Wilson, R.M., Reichman, E.J.: 1999, A synthesis of solar cycle prediction techniques. J. Geophys. Res. 104, 375. DOI.

Hoeksema, J.T.: 2010, Evolution of the large-scale magnetic field over three solar cycles. In: Kosovichev, A.G., Andrei, A.H., Rozelot, J.-P. (eds.) Solar and Stellar Variability: Impact on Earth and Planets, IAU Symposium 264, 222. DOI. ADS.

Howard, R.: 1977, Large-scale solar magnetic fields. Annu. Rev. Astron. Astrophys. 15, 153. DOI. ADS.

Leighton, R.B.: 1969, A magneto-kinematic model of the solar cycle. Astrophys. J. 156, 1. DOI. ADS.

Li, K.J., Yun, H.S., Gu, X.M.: 2001, On long-term predictions of the maximum sunspot numbers of solar cycles 21 to 23. Astron. Astrophys. 368, 285. DOI.

McNish, A.G., Lincoln, J.V.: 1949, Prediction of sunspot numbers. Eos Trans. AGU 30, 673. DOI. ADS.

Muñoz-Jaramillo, A., Nandy, D., Martens, P.C.H.: 2009, Helioseismic data inclusion in solar dynamo models. Astrophys. J. 698, 461. DOI. ADS.

Muñoz-Jaramillo, A., Sheeley, N.R., Zhang, J., DeLuca, E.E.: 2012, Calibrating 100 years of polar faculae measurements: implications for the evolution of the heliospheric magnetic field. Astrophys. J. 753, 146. DOI. ADS.

Ohl, A.I., Ohl, G.I.: 1979, A new method of very long-term prediction of solar activity. In: Donnelly, R. (ed.) Solar-Terrestrial Predictions Proceedings 2, 258. NOAA/Space Environment Laboratory. ADS.

Parker, E.N.: 1977, The origin of solar activity. Annu. Rev. Astron. Astrophys. 15, 45. DOI. ADS.

Pesnell, W.D.: 2008, Predictions of Solar Cycle 24. Solar Phys. 252, 209. DOI. ADS.

Pesnell, W.D.: 2014, Predicting Solar Cycle 24 using a geomagnetic precursor pair. Solar Phys. 289, 2317. DOI. ADS.

Pesnell, W.D.: 2016, Predictions of Solar Cycle 24: how are we doing? Space Weather 14, 10. DOI. ADS.

Priyal, M., Banerjee, D., Karak, B.B., Muñoz-Jaramillo, A., Ravindra, B., Choudhuri, A.R., Singh, J.: 2014, Polar network index as a magnetic proxy for the solar cycle studies. Astrophys. J. Lett. 793(1), L4. DOI.

Schatten, K.H.: 1990, A solar cycle timing predictor-the latitude of active regions. Solar Phys. 125, 185. DOI. ADS.

Schatten, K.H.: 2005, Fair space weather for solar cycle 24. Geophys. Res. Lett. 32, L21106. DOI.

Schatten, K.H., Myers, D.J., Sofia, S.: 1996, Solar activity forecast for Solar Cycle 23. Geophys. Res. Lett. 23, 605. DOI. ADS.

Schatten, K.H., Pesnell, W.D.: 1993, An early solar dynamo prediction: cycle $23 \sim$ cycle 22. Geophys. Res. Lett. 20, 2275. DOI. ADS.

Schatten, K.H., Sofia, S.: 1987, Forecast of an exceptionally large even-numbered solar cycle. Geophys. Res. Lett. 14, 632. DOI. ADS.

Schatten, K.H., Scherrer, P.H., Svalgaard, L., Wilcox, J.M.: 1978, Using Dynamo Theory to predict the sunspot number during Solar Cycle 21. Geophys. Res. Lett. 5, 411. DOI.

Sheeley, N.R., Wang, Y.-M., Harvey, J.W.: 1989, The effect of newly erupting flux on the polar coronal holes. Solar Phys. 119, 323.

Suggs, R.J.: 2013, Future solar activity estimates for use in prediction of space environmental effects on spacecraft orbital lifetime and performance. Technical Report, NASA, Marshall Space Flight Center. File dated June 2013. http://sail.msfc.nasa.gov/current_solar_report/CurRpt.pdf.

Svalgaard, L., Cliver, E.W., Kamide, Y.: 2005, Sunspot Cycle 24: smallest cycle in 100 years? Geophys. Res. Lett. 32, 1104. DOI. ADS.

Tapping, K.F.: 2013, The $10.7 \mathrm{~cm}$ solar radio flux $\left(\mathrm{F}_{10.7}\right)$. Space Weather 11, 394. DOI. ADS.

Waldmeier, M.: 1939, Über die Struktur der Sonnenflecken. Astron. Mitt. Eidgenöss. Sternwarte Zür. 14, 439. ADS. 
Waldmeier, M.: 1955, Ergebnisse und Probleme der Sonnenforschung, 2. erweiterte aufl. edn. Geest \& Portig, Leipzig. ADS.

Zhao, J., Kosovichev, A.G., Bogart, R.S.: 2014, Solar meridional flow in the shallow interior during the rising phase of Cycle 24. Astrophys. J. Lett. 789, L7. DOI. ADS.

Zhao, J., Bogart, R.S., Kosovichev, A.G., Duvall, T.L. Jr., Hartlep, T.: 2013, Detection of equatorward meridional flow and evidence of double-cell meridional circulation inside the Sun. Astrophys. J. Lett. 774, L29. DOI. ADS. 